\title{
ANÁLISE DE VIABILIDADE ECONOMICA DA IMPLANTAÇÃO DE UM SISTEMA FOTOVOLTAICO EM UMA MICROEMPRESA NO ESTADO DO AMAZONAS
}

\author{
ANALYSIS OF ECONOMICAL FEASIBILITY OF THE IMPLANTATION OF A \\ PHOTOVOLTAIC ENERGY SYSTEM IN A MICRO-COMPANY IN THE AMAZONIAN STATE
}

Recebido em: 01 nov. 2019

Aprovado em: 30 mar. 2020

Versão do autor aceita publicada online: 30 mar. 2020

Publicado online: 19 maio 2021

Como citar esse artigo - American Psychological Association (APA):

Freires, V. de M., Almeida, E. S., \& Freires, V. de M. (2021, jul./set.). Análise de viabilidade econômica da implantação de um sistema fotovoltaico em uma microempresa no estado do Amazonas. Exacta, 19(3), 693-705. https://doi.org/10.5585/exactaep.2021.15956.

Submeta seu artigo para este periódico $\beta$

Dados Crossmark 


\title{
ANÁLISE DE VIABILIDADE ECONOMICA DA IMPLANTAÇÃO DE UM SISTEMA FOTOVOLTAICO EM UMA MICROEMPRESA NO ESTADO DO AMAZONAS
}

\author{
ANALYSIS OF ECONOMICAL FEASIBILITY OF THE IMPLANTATION OF A PHOTOVOLTAIC ENERGY SYSTEM \\ IN A MICRO-COMPANY IN THE AMAZONIAN STATE
}

\section{iD Vitoria de Melo Freires ${ }^{1}$ \\ (iD) Ely Sena de Almeida ${ }^{2}$ \\ ID Viviane de Melo Freires ${ }^{3}$}

${ }^{1}$ Estudante de Engenharia de Produção

Universidade Federal do Amazonas - UFAM.

Manaus, Amazonas - Brasil.

vikdmf@gmail.com

${ }^{2}$ Mestre em Engenharia de Produção

Universidade Federal do Amazonas - UFAM

Manaus, Amazonas - Brasil.

elysenaa@ufam.edu.br

${ }^{3}$ Engenheira de Produção

Universidade Federal do Amazonas - UFAM.

Manaus, Amazonas - Brasil.

vivimelofreires@gmail.com

Recebido em: 01 nov. 2019

Aprovado em: 30 mar. 2020
Resumo: O presente artigo tem como objetivo analisar a viabilidade da implantação do sistema fotovoltaico em uma microempresa localizada no estado do Amazonas, assim também como analisar os seus benefícios para tal empresa com esse sistema. Tal estudo visa ajudar microempresários na tomada de decisão em relação a adoção do sistema fotovoltaico em seus investimentos e tornar mais conhecido o grande potencial do Brasil em relação a energia solar. $\mathrm{O}$ atual investimento do Brasil em hidrelétricas causa preocupações em relação aos impactos ao meio ambiente, desse modo torna-se necessário a pratica da Responsabilidade social, onde deve ter maior preocupação com o meio ambiente para gerações futuras. O presente estudo mostrou um desempenho satisfatório em relação ao sistema fotovoltaico, pois, além de ser uma energia mais limpa, mostrou através dos gráficos e resultados obtidos um grande desempenho e diminuição nos custos com energia, o que é um problema atual da microempresa.

Palavras-chave: Sistema fotovoltaico. Responsabilidade social. Diminuição de custos.

Abstract: This article aims to analyse the viability of photovoltaic system deployment in an enterprise located in the State of Amazonas, as well as analyze their benefits to such company with this system. This study aims to help microentrepreneurs in decision-making in relation to adoption of photovoltaic system on their investments and making better known the great potential of Brazil in relation to solar energy. The current Brazil's investment in dams cause concerns regarding environmental impacts, thereby becomes necessary the practice of corporate social responsibility, where it should have greater concern for the environment for future generations. The study showed a satisfactory performance in relation to the PV system, because, besides being a cleaner energy, has shown through graphs and results achieved a great performance and reduction in energy costs, which is a current problem microenterprise.

Key words: Photovoltaic system. Social responsibility. Reducing costs. 
1 Introdução

Atualmente as usinas hidrelétricas não mostram um desempenho satisfatório, tanto em relação ao desenvolvimento sustentável, quanto em relação a seu investimento que em alguns casos geram grandes dividas para sua construção, como exemplo foi o caso da Usina Hidrelétrica de Santo Antônio e a Hidrelétrica de Balbina, que produz muito abaixo do esperado. Além disso a energia elétrica produzida por elas tem altos custos e com o passar dos anos a sua tarifa de energia aumenta cada vez mais, podendo mudar de região para região.

Segundo dados do jornal Em Tempo, publicado em 30/03/2019, a população do Amazonas paga a energia elétrica mais cara do país (EM TEMPO, 2019). O quilowatt hora (Kwh) é de $R \$ 1,07$ enquanto a média nacional é de $\mathrm{R} \$ 0,87$. O motivo é a ineficiência da Usina de Balbina, pois é insuficiente para suprir a demanda de Manaus, sendo necessário a compra de outros fornecedores. A construção da Hidrelétrica de Balbina teve como consequências o alagamento e formação de um lago com cerca com cerca de 2.360 quilômetros quadrados, com potencial de 250 Megawatts de energia. Comparando com uma Hidrelétrica mais eficiente, a hidrelétrica de Tucuruí, no Pará, teve uma alagação de área semelhante e produz cerca de $8.370 \mathrm{MW}$ de energia.

Em contra partida, a energia solar já se mostra com grande potencial para geração de energia elétrica, são várias as vantagens desse sistema, como por exemplo, uma alternativa mais sustentável para geração de energia e com o tempo ela se torna auto sustentável, ou seja, depois de um tempo se recupera o investimento inicial e a energia se torna praticamente gratuita, ela produz o necessário para a demanda do imóvel.

Além dessa visão geral das vantagens desse sistema, há também as vantagens em relação a empresas, como vai ser estudado nesse artigo, pois muitas empresas têm custos elevados com a energia elétrica distribuída pelo sistema local porém essas não sabem como diminuir esse custo ou não percebem o potencial de uma energia alternativa para diminuir esse custo. Desse modo a energia solar fotovoltaica se torna uma grande possibilidade para diminuição desses custos pois é um bom investimento com grandes vantagens em relação a custo em médio prazo. O custo de aquisição inicial é um pouco elevado para algumas empresas e pessoas físicas porém atualmente já tem propostas das concessionárias que facilitam a compra desse sistema de energia no qual se pode pagar parcelas parecidas com as contas mensais de energia que se paga para a distribuição de energia regional. Ou seja, não terá grande impacto no orçamento e não precisará ter um valor inicial tão grande para sua compra.

Artigos como esse são de grande importância tanto para o meio acadêmico como para empresas que, podem tomar esse artigo como base para tomada de decisão em um investimento em um sistema solar fotovoltaico. Além disso, é de suma importância para tornar a energia solar 
fotovoltaica mais conhecida e mostrar que não é só a classe alta que pode obtê-la. Atualmente esta realidade mudou e está mais acessível adquirir um sistema renovável de energia em sua casa, além de reduzir custos com energia e ter um país mais sustentável.

O estudo desenvolvido iniciou com uma pesquisa explanatória, que buscou informações quanto aos sistemas de energia solar possíveis para implantação no Brasil, para assim delimitar o tema, objetivos e justificativa. Em seguida foi realizado a pesquisa bibliográfica para estudos mais recentes na área para assim tomar como base no presente projeto de pesquisa e saber a atual realidade do Brasil e do estado do Amazonas em relação a energia elétrica. E, por fim, uma pesquisa investigativa pois utiliza o levantamento de dados e analise de arquivos. O estudo delimitou-se a uma empresa microempresa, para agilizar e obter de forma mais rápida os resultados e analisar a hipótese inicial do trabalho. A escolha da mesma se deu por conta da facilidade de obter os dados e facilidade em propor tal solução do problema para os responsáveis da empresa. Tal empresa está localizada no município de Presidente Figueiredo.

A hipótese é que a energia solar fotovoltaica é uma solução para diminuição de custos com energia de uma microempresa, além também de não depender da distribuição de energia local que sofre problemas com a instabilidade da energia elétrica. O custo de aquisição, payback e irradiação solar local são as variáveis dependentes. As variáveis independentes são: área do estabelecimento, consumo médio mensal de energia, e clima. Esta pesquisa é de carácter sustentável e econômico, no qual o tema escolhido está relacionado a solução do problema energético para microempresas do Amazonas assim também relacionado a Responsabilidade Social Empresarial e diminuição de custos da organização.

A abordagem mista foi escolhida para esse trabalho pois dá a liberdade para o pesquisador em poder usar os dois métodos, qualitativa e quantitativa, para solucionar o problema de pesquisa. A qualitativa se preocupa em obter informações e interpretar o ambiente em que a problemática acontece leva em consideração a importância da concepção da realidade organizacional. Com isso, a abordagem qualitativa se deu para analisar o ambiente e a problemática da empresa. A quantitativa utiliza hipóteses, derivadas de um referencial teórico, associadas às variáveis e captura as evidências da pesquisa por meio da mensuração das variáveis. Dessa forma, será utilizado as variáveis, dados quantitativos para observar a hipótese inicial para resolução dos problemas analisados.

\section{Referencial teórico}

\subsection{Energia Renovável}

Segundo Villalva e Gazoli (2012), o Sol é a principal fonte do nosso planeta. A superfície da terra recebe por ano uma quantidade de energia solar na forma de luz e calor e apenas uma parcela dela é 
aproveitada. Ainda assim, praticamente toda a energia usada pelo ser humano tem origem no sol, são poucas as exceções que não cabem nessa afirmação.

Os mesmos autores afirmam que as fontes renováveis de energia são as que são consideradas inesgotáveis para os padrões humanos. Ou seja, ela pode ser usada e nunca irá se acabar pois sempre se renovam. Algumas dos principais exemplos de energias renováveis são: Hidrelétrica, eólica, solar, oceânica, geotérmica e da biomassa.

Outro conceito importante abordado pelos autores Villalva e Gazoli (2012), é o conceito de fontes limpas de energia que normalmente é associada as fontes renováveis, pois em comparação com os combustíveis fosseis eles praticamente não originam resíduos ou emissões de poluentes. Entretanto ainda sim elas não podem ser consideradas limpas pois provocam alterações no meio ambiente e produz impactos de maior ou menor relevância. É importante ressaltar a explicação dos autores:

"A instalação de geradores eólicos provoca a morte de pássaros, produz ruídos audíveis e modifica as paisagens. Na fabricação de geradores eólicos e células fotovoltaicas empregam-se componentes tóxicos. As usinas solares térmicas empregam fluidos tóxicos e sua instalação ocupa grandes áreas e afeta habitats naturais. A construção de usinas hidrelétricas requer grandes quantidades de matériaprima e energia e a formação de represas inunda enormes áreas e altera irreversivelmente o ambiente no seu entorno." (Villalva \& Gazoli, 2012, . 16-17).

Isso mostra que nenhuma energia pode ser considerada totalmente limpa, porem pode ser considerada mais limpa em comparação a outra, levando em consideração os seus impactos no meio ambiente.

\subsubsection{Energia Solar Fotovoltaico}

A Energia solar pode ser dividida em duas, a energia solar térmica e a energia solar Fotovoltaica. No livro Energia Solar fotovoltaica, os autores Villalva e Gazoli (2018), explicam melhor a diferença entre esses dois sistemas. A energia solar térmica utiliza as placas solares para aquecer a agua utilizando diretamente o calor do sol. Já a energia solar fotovoltaica produz eletricidade através do efeito fotovoltaico, que consiste na conversão direta da luz solar em energia elétrica (Villalva \& Gazoli, 2018).

“Diferentemente dos sistemas solares térmicos, que são empregados para realizar aquecimento ou para produzir eletricidade a partir da energia térmica do sol, os sistemas fotovoltaicos têm a capacidade de captar diretamente a luz solar e produzir corrente elétrica. Essa corrente é coletada e processada por dispositivos controladores e conversores, podendo ser armazenada em baterias ou utilizada diretamente em sistemas conectados à rede elétrica." (Villalva \& Gazoli, 2018, p. 21). 
Na figura 1 é demonstrado de maneira ilustrativa a diferença dos sistemas energéticos citados.

\section{Figura 1}

\section{Diferença entre Energia Solar Térmica e Fotovoltaica}

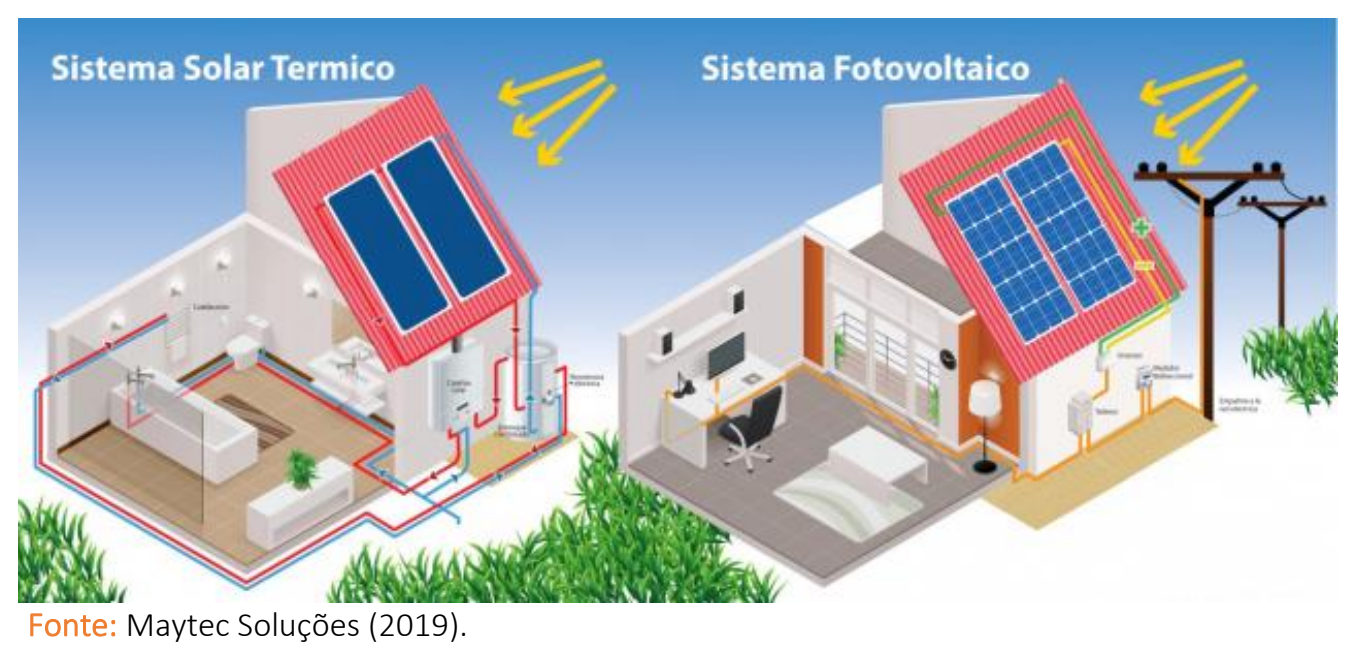

A imagem mostra a diferença entre a ligação das placas solares térmicas e fotovoltaicas. A Térmica liga somente a parte hidráulica da casa, já a fotovoltaica se liga a parte elétrica da casa.

A energia solar fotovoltaica pode ser dividida em 2 tipos, os sistemas isolados ou autônomos, "[...] são empregados em locais não atendidos por rede elétrica" (Villalva \& Gazoli, 2018, p.99), e o outro são os sistemas fotovoltaicos conectados à rede elétrica, "[...] conectado à rede elétrica opera em paralelismo com a rede de eletricidade." (Villalva \& Gazoli, 2018, p. 149). Para melhor entendimento do sistema solar fotovoltaico segue a Figura 2 : 
Figura 2

Funcionamento do sistema Fotovoltaico

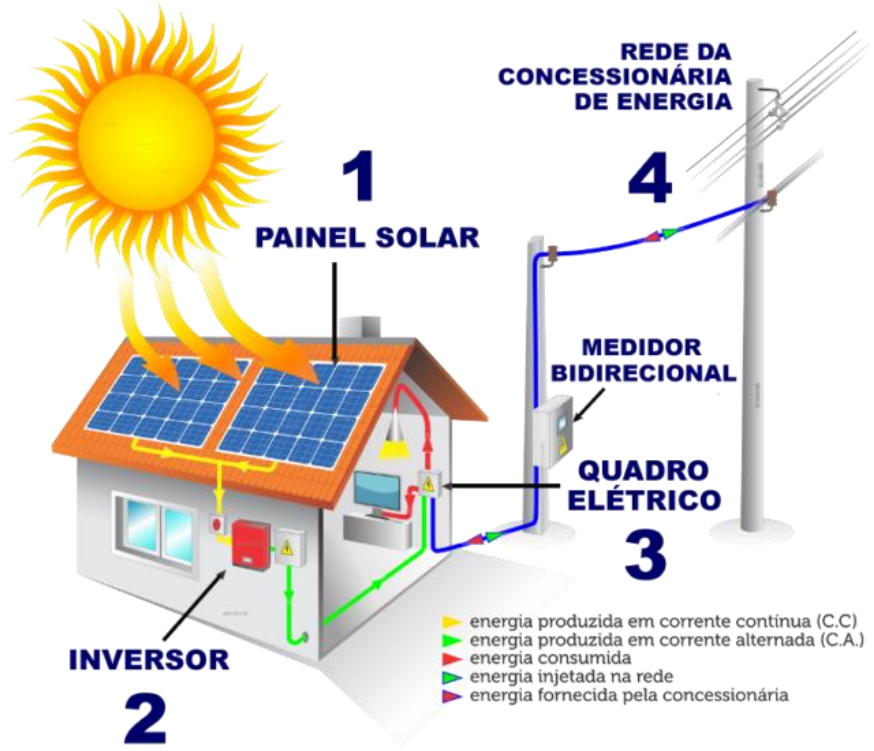

Fonte: Maytec Soluções (2019).

\subsection{Payback}

O Payback é uma palavra em inglês que significa "retorno", se refere ao tempo em que se recupera o investimento inicial. O Payback, ou tempo de recuperação do capital, consiste na mensuração do número de anos requeridos para a recuperação de um dado investimento inicial, ou seja, este método mede o tempo necessário para que a soma das quantias anuais seja igual ao investimento original (Casarotto \& Kopittke, 2010).

De acordo com Gitman (2010) o período de payback é um instrumento utilizado para avaliar investimentos. Denomina-se payback como o tempo necessário para a empresa recuperar seu investimento inicial em um projeto calculado a partir de seus fluxos de caixa de entrada. Este período de payback máximo aceitável é definido pela administração da empresa, que tem como base investimentos anteriores, rentabilidade pretendida, entre outros fatores.

\section{Procedimentos metodológicos}

\subsection{Objeto de estudo}

O presente artigo irá apresentar a viabilidade da implantação de um sistema fotovoltaico em uma microempresa localizada no Estado do Amazonas. Com os dados coletados da microempresa e do ambiente onde está localizada foi simulado qual seria o investimento nas placas solares assim também como o payback. Tal ação propõe a solução para diminuição de custos com energia da microempresa 
assim também como ter uma energia alternativa para os casos de falta de energia do sistema convencional que ocorrem na região estudada.

A escolha dessa proposta foi definida pelos altos gastos com energia da microempresa, levando em consideração também o alto potencial do sistema fotovoltaico para a região. A partir disso realizouse a simulação de investimento em um simulador online.

Por se tratar de uma microempresa privada escolhida para o estudo, iremos mencioná-la como empresa Alfa e por questão de segurança da informação foram avaliados seus métodos de coleta de dados e transcritos de forma que não haja maleficio algum para a empresa. De todo modo, vale a pena ressaltar que tal empresa está localizada no município de Presidente Figueiredo.

\subsection{Procedimentos de coleta de dados}

O levantamento de dados foi realizado através da solicitação da planta do estabelecimento e o levantamento de histórico de energia através do site da Manaus Energia para assim analisar a área disponível para as placas solares e consumo médio mensal de energia para simular a quantidade necessária de placas solares.

Foi coletado também dados do clima local, como temperatura média local e a média de irradiação que incide na região, pois são dados importantes para saber se o sistema fotovoltaico será realmente uma solução para tal problema estudado.

Para a coleta de dados foi dedicado um total de 4 dias de trabalho, iniciado no dia 5 de junho a 9 de junho. A empresa tem um horário de funcionamento de $8 \mathrm{~h}$ da manhã ás $8 \mathrm{~h}$ da noite, tendo um intervalo para o almoço de $12 \mathrm{~h}$ as $14 \mathrm{~h}$, totalizando assim $10 \mathrm{~h}$ de trabalho por dia na microempresa. Como a empresa está localizada no município de Presidente Figueiredo, consideramos, nesses 4 dias de coleta, as horas de viagem para locomoção até o local a ser estudado. Desse modo, de 40 horas que totalizam o horário de trabalho desses 4 dias, foram considerados 4 h a menos que representam a locomoção de ida e volta entre Presidente Figueiredo e Manaus, totalizando assim 36h de coletas de dados.

$\mathrm{Na}$ coleta de todos os dados foram necessários 2 pesquisadores, e de forma igualitária foi definido quais dados seriam coletados por cada um deles dentre os seguintes tópicos: consumo mensal de energia da empresa Alfa, planta do imóvel, clima local, irradiação solar e Tarifa de energia com impostos (R\$/kWh). Todos esses dados foram agrupados em planilhas no Excel. 


\subsection{Procedimentos de análise de dados}

Para a análise dos dados utilizou-se uma calculadora online para simular os investimentos. Todos os dados coletados foram diretamente ligados aos dados necessários para fazer os cálculos de todo o sistema fotovoltaico.

Uma das calculadoras solar que foi usada nesse artigo é da Ventusolar. A Ventusolar é especializada em projetos e instalações de sistemas de geração de energia fotovoltaica e eólica. Eles auxiliam as empresas ou residências a reduzir suas contas de lua utilizando os sistemas fotovoltaicos ou eólico conectados à rede de energia elétrica ou isolados. Esta empresa cuida de todo o processo, desde a avaliação financeira até mesmo a instalação dos equipamentos e a regularização junto a concessionaria de energia. Tudo isso facilita ao contratante na hora de tomar a decisão e implantar o sistema em seu imóvel.

A calculadora solar da Ventusolar solicita dados como Estado, cidade, consumo mensal de energia, tipo de ligação elétrica etc. A partir desses dados a própria calculadora fornece dados como, quantidade de placas solares indicadas para o imóvel, fornece o gráfico de consumo médio mensal de energia em comparação a energia gerada por mês pelo sistema fotovoltaico, logo em seguida um gráfico mostrando a economia com o passar dos meses com a implantação do sistema e nesse mesmo gráfico há duas linhas de comparação entre a conta de luz sem o sistema fotovoltaico e com o sistema também. Ao final é disponibilizado a tabela de viabilidade econômica financeira, no qual são fornecidos os seguintes dados: economia mensal na conta de luz, economia percentual mensal e payback.

Para fazer a simulação na Ventusolar foi necessário o preenchimento da tabela com os seguintes dados: Nome, E-mail, Telefone, Estado, Cidade, distribuidora de energia, inclinação dos paneis, tipo de ligação elétrica e tipo de imóvel. Em uma segunda tabela foi preenchido os dados de consumos mensais de energia por mês ( $\mathrm{kWh} / \mathrm{mês}$ ), sendo preenchido os 12 meses de consumo, de janeiro a dezembro.

\section{Resultados}

A Calculadora Solar Ventusolar possibilitou a analisa dos dados e resultados de mais variadas formas para a análise. Após o preenchimento dos quadros 1 e 2, obteve-se os seguintes resultados. Os dados da tabela 2 é referente ao consumo mensal dos últimos 12 meses. 
Quadro 1

Características Técnicas do Sistema Fotovoltaico

\begin{tabular}{|l|l|}
\hline \multicolumn{2}{|c|}{ CARACTERISTICAS TÉCNICAS } \\
\hline $\begin{array}{l}\text { Quantidade Ideal de placas fotovoltaicas } \\
\text { (Calculado de acordo com historico de consumo) }\end{array}$ & \\
\hline Quantidade desejada de placas fotovoltaicas & \\
\hline Potência da placa fotovoltaica & $330 \mathrm{Wp}$ \\
\hline Potência do Kit Fotovoltaico $(\mathrm{kWp})$ & $4,95 \mathrm{kWp}$ \\
\hline Área necesária ( $\left.\mathbf{m}^{2}\right)$ & $30,9 \mathrm{~m}^{2}$ \\
\hline Tarifa de energia com impostos $(\mathbf{R S} / \mathrm{kWh})$ & 1,08 \\
\hline
\end{tabular}

Fonte: Ventusolar (2019).

Este quadro mostra a quantidade de placas fotovoltaicas indicadas pela calculadora solar para suprir a demanda da microempresa Alfa, a partir dos dados que foram colocados e preenchidos na simulação. Dessa forma, tomou-se a sugestão de 15 placas solares para fazer a simulação. Vale a pena ressaltar que, de acordo com a planta do imóvel obtida pelos pesquisadores, é possível a implantação dessas 15 placas que ocupariam 30,9 metros quadrados no telhado do imóvel.

Tabela 1

Consumo Geral vs Energia Gerada (Sistema Fotovoltaico)

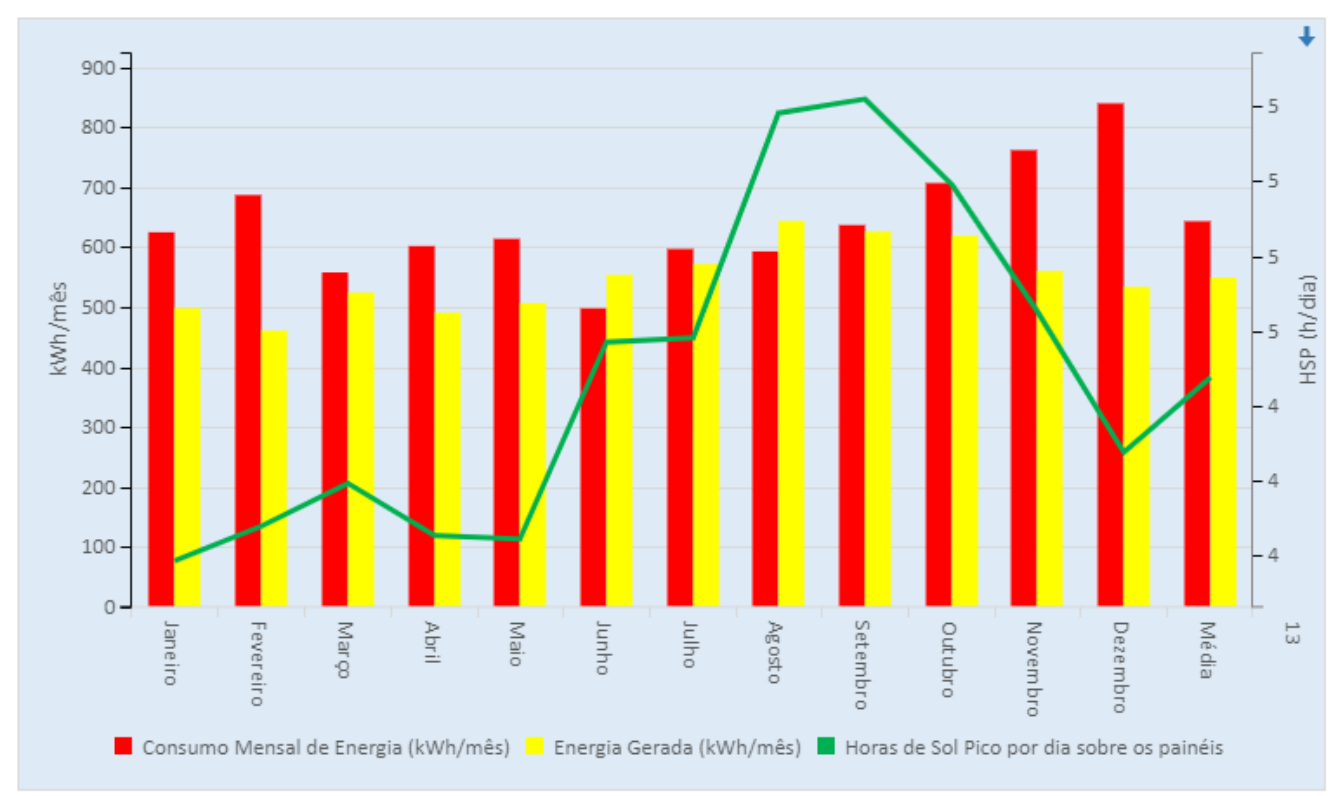

Fonte: Ventusolar (2019). 
O gráfico acima mostra desempenho em amarelo ao adotar as 15 placas solares para a microempresa Alpha, de tal modo que a energia gerada pelo sistema fotovoltaico chega bem próximo ao consumo de energia do imóvel, com isso, diferença entre eles mostra a quantidade em Kwh que teria que ser pago ao sistema de energia convencional da região.

\section{Grafico 2}

\section{Economia de luz}

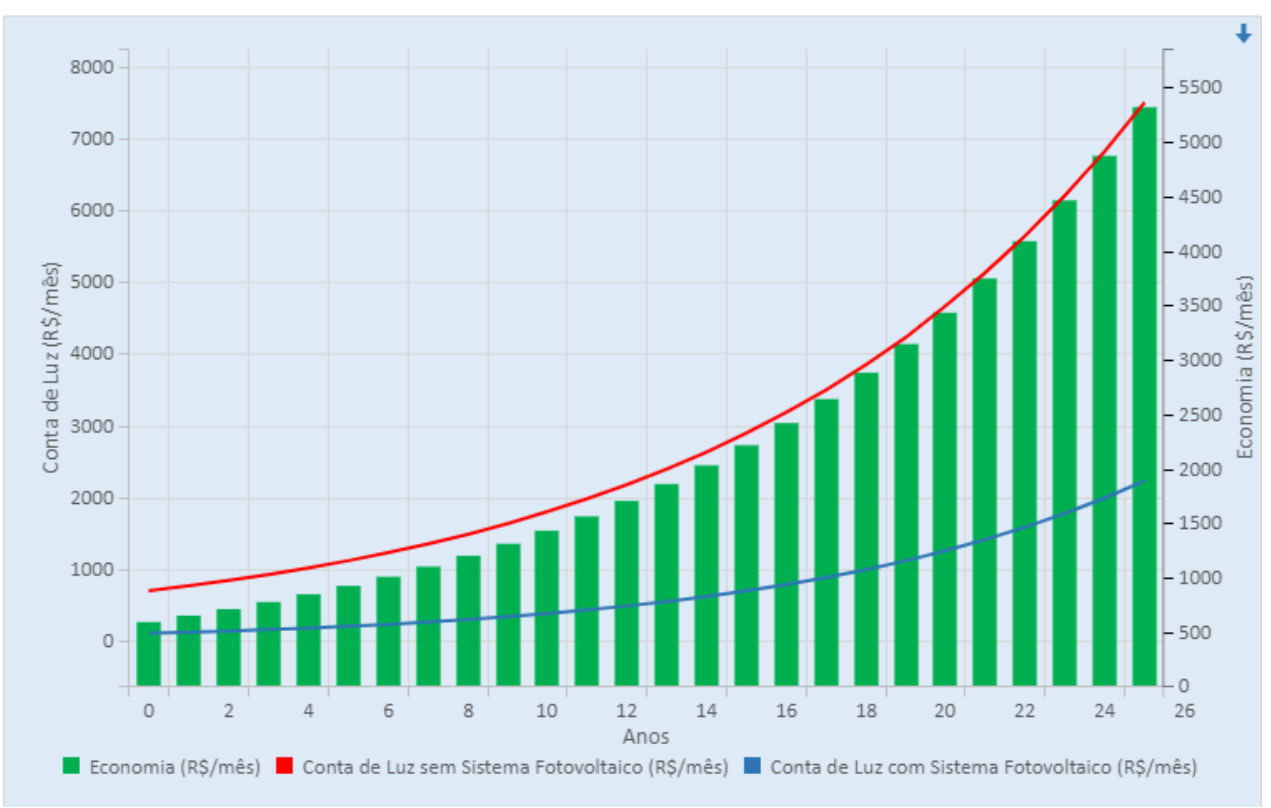

Fonte: Ventusolar (2019).

O gráfico 2 mostra a economia em Reais que seria feita por mês após a adoção do sistema fotovoltaico. A linha vermelha mostra a conta de luz sem o sistema fotovoltaico já a linha azul mostra a conta de luz com o sistema fotovoltaico e nota-se uma diferença significativa entre elas. 


\section{Quadro 2}

\section{Viabilidade Econômica Financeira}

\begin{tabular}{|l|l|}
\hline \multicolumn{2}{|c|}{ VIABILIDADE ECONÔMICO FINANCEIRA } \\
\hline Economia mensal na conta de luz & $\mathrm{R} \$ 591,12$ \\
\hline Economia Percentual Mensal & $85,36 \%$ \\
\hline $\begin{array}{l}\text { Payback } \\
\text { (O Payback é o tempo que levará para que o seu investimento retorne ao seu } \\
\text { bolso em forma de economia) }\end{array}$ & 3 anos e 4 meses \\
\hline $\begin{array}{l}\text { TIR em } 10 \text { anos } \\
\text { (A TIR de um investimento pode ser entendida, para fins comparativos, como a } \\
\text { taxa de juros de uma aplicação financeira.) }\end{array}$ & $46,99 \%$ a.a. \\
\hline $\begin{array}{l}\text { Custo Nivelado da Energia } \\
\text { (Este valor é quivalente à tarifa de energia. } \\
\text { Compare com o valor que vocé paga atualmente) }\end{array}$ & $0,30 \mathrm{R} \$ / \mathrm{kWh}$ \\
\hline
\end{tabular}

Fonte: Ventusolar (2019).

O último quadro mostra o payback de investimento em 15 placas solares que foi indicado pela própria calculadora solar. Mostra também a economia de quase 600 reais na conta de luz ao mês.

\section{Discussões}

Em todo o mundo torna-se necessário a busca pelo desenvolvimento sustentável e, é claro, buscar a responsabilidade social para assim garantir uma boa vida para as futuras gerações, contudo toda empresa deve também buscar formas de diminuir custos e aumentar seus lucros. A proposta principal desse artigo era buscar tudo isso citado e com esse estudo confirmou-se a proposta inicial de resolução do problema, é claro que deve ser estudado o payback pela microempresa alpha, no qual deve ser estipulado pela administração o tempo máximo de retorno; mas o sistema fotovoltaico mostrou-se eficaz para a diminuição de custos da empresa, uma vez que, diminui cerca de $85 \%$ da conta de luz, e ajudaria muito na problemática enfrentada pela empresa por conta de alto custo com energia. Por ser uma microempresa, as possibilidades de diminuição de custos em outras áreas são muito pequenas e então esses $85 \%$ de economia na conta de luz já seria grande contribuição para o lucro da empresa.

Após toda a análise do desempenho do sistema fotovoltaico, é importante analisar a viabilidade do mesmo. Para a tomada de decisão dos proprietários da microempresa, faz-se necessário a análise de vários fatores, a princípio é necessário simular se o sistema é realmente eficaz para tal região, e isso foi comprovado pelos gráficos anteriores, mas isso não torna suficiente. A tabela acima mostra a quantidade em reais que seria economizado em média por mês e o percentual de $85,36 \%$ de economia na conta de luz, como a empresa Alpha tem problemas com o alto custo de conta de energia, essa porcentagem de quase $90 \%$ seria de grade benefício para a empresa. 
O tempo estimado para o retorno do investimento é de 3 anos e 4 meses, e para a tomada de decisão, o payback é muito importante. Caso o payback seja usado para a tomada de decisão, deve ser levado em consideração os seguintes critérios: Se o período de retorno do investimento for menor que o período máximo aceitável de recuperação, então o projeto será aceito; caso o período de retorno for maior que o aceitável para recuperação, então o projeto será recusado. O período aceitável de recuperação é definido pela administração da empresa, desse modo é necessário fazer uma segunda pesquisa com os administradores da microempresa Alpha para analisar o tempo aceitável por eles para o retorno do investimento.

\section{Conclusões}

O objetivo principal desse artigo era analisar a viabilidade da implantação do sistema fotovoltaico na microempresa Alpha e analisar os benefícios da implantação do mesmo, desse modo, ao final desse artigo, o objetivo foi alcançado de modo satisfatório através da análise de todos os gráficos e dados obtidos pela Calculadora solar, no qual, mostrou muitos benefícios na adoção do sistema e mostrou também toda a parte financeira, dado muito importante para a tomada de decisão, pois mostra a economia mensal e retorno do investimento nessa hipótese de melhoria. Tal tema é de grande importância para o Brasil pois o país tem grande potencial para o sistema fotovoltaico, assim também como solucionar problemas com a falta de energia e outros problemas relacionados ao mesmo como o elevado custo da energia elétrica. Desse modo, tornasse necessário mais estudos na área de energia solar de modo que esse tipo de sistema se torne mais conhecido e adquira mais adeptos.

Esse artigo fortalece a teoria de que a energia solar é uma ótima solução para um pais mais sustentável e econômico, mostrando assim a sua grande contribuição para a parte teórica do estudo. Outra grande contribuição desse artigo em relação a pratica é o resultado satisfatório mostrado nesse estudo para a microempresa estudada, provando assim que é um bom investimento e outros imóveis podem utilizar tal tecnologia da energia solar.

Este artigo limitou-se apenas a uma microempresa, porém, pode ser estudada em outras empresas e até mesmo nas faculdades, então, fica como sugestão para trabalhos futuros, um estudo de análise de viabilidade e desempenho de um sistema fotovoltaico em laboratórios de faculdades, como uma forma de suprir a demanda do local e torna-lo mais sustentável, sem esquecer, é claro, na diminuição da conta de luz e resolver o problema de cancelamento de aulas por conta da falta de energia do sistema convencional fornecido para a região.

\section{Referências}

Calculadora Solar (2018) [programa de computador] . Florianópolis, Santa Catarina: Ventusolar. 
Casarotto Filho, Nelson; Kopittke, Bruno Hartmut (2010). Análise de Investimentos: Matemática Financeira, Engenharia Econômica, Tomada de Decisão, Estratégia Empresarial. (11 ed.) São Paulo: Atlas.

Em tempo (2019). Amazonas tem a tarifa de energia mais cara do país. (2019). Recuperado em 24 abril, 2019, de: https://d.emtempo.com.br/economia/142022/amazonas-tem-a-tarifa-deenergia-mais-cara-do-pais

Gitman, L. J.(2004) Princípios de administração financeira. (10. ed.) São Paulo: Pearson Addison Wesley.

Maytecsolucoes (2019). Como funciona a energia solar fotovoltaica. Recuperado em 12 maio, 2019, de: https://www.maytecsolucoes.com.br/energia-solar

Villalva, M. G.; Gazoli, J. R.(2018) Energia Solar Fotovoltaica (2. ed.) Tatuapé: Érica Ltda. 